\title{
Barrieren der Markenführung bei Winzergenossenschaften
}

\author{
Torsten Proschwitz und Jon H. Hanf \\ Hochschule Geisenheim University, 65366 Geisenheim, Germany
}

\begin{abstract}
Markenbildung gewinnt in der Weinwirtschaft immer mehr an Bedeutung. Um sich im Weinmarkt, einem Markt mit hoher Wettbewerbsintensität durchzusetzen, bedarf es der Produktdifferenzierung. Da Weine in der Regel regionale Produkte sind, deren Qualität nach dem Verständnis der meisten Konsumenten ausschließlich durch Rebsorte, Herkunft und Jahrgang geprägt wird, eignet sich der Ansatz der Markenführung als Differenzierungsstrategie um ein Markenimage im Kopf der Verbraucher aufzubauen, welches hohe Relevanz für das Kaufverhalten hat. Genossenschaften machen mit rund einem Drittel der Weinerzeugung einen erheblichen Teil am Markt aus. Aufgrund der Strukturen einer Genossenschaft kommt es jedoch zu Barrieren der Markenführung, welche langfristige strategische Investitionen in den Aufbau einer Marke nicht zulassen. Neue Institutionenökonomie wird in der agrarökonomischen Forschung häufig als Erklärung dafür herangezogen, dass Landwirte in Genossenschaften auf die Maximierung des Auszahlungspreises fokussiert sind und daher strategische Investitionen ablehnen. Da die Winzergenossenschaften auf die Zusammenarbeit mit ihrer Mitgliederbasis auf Grund des Demokratieprinzips angewiesen sind, können folglich notwendige strategische Investitionen unterbleiben. Dieses Paper liefert Erklärungsansätze für strategische Defizite in genossenschaftlichen Verarbeitungsunternehmen der Weinindustrie und versucht Lösungsansätze aufzuzeigen.
\end{abstract}

In the wine industry branding is becoming increasingly important. In order to succeed in the wine market a market with intense competition - product differentiation is necessary. Wine is usually a product which quality is influenced by the understanding of most consumers exclusively by grape variety, origin and vintage: Opposite the approach of brand management is a differentiation strategy around a brand image in the consumer mind. Cooperatives - with approximately one third of the wine production - are a significant part of the German market. However, due to the governance structures of cooperatives there are barriers of branding, which does not allow long-term strategic investments in building a brand. Based on New Institutional Economics this paper provides explanations for these barriers and tries to identify solutions.

Branding gagne dans l'industrie du vin est de plus en plus importante. Pour réussir sur le marché du vin, un marché où la concurrence intense, il nécessite la différenciation des produits. Depuis vins généralement des produits régionaux dont la qualité est influencée par la compréhension de la plupart des consommateurs exclusivement par cépage, l'origine et vintage, l'approche de la gestion de la marque est une stratégie de différenciation autour d'une image de marque dans l'esprit des consommateurs mis en place, qui sont très pertinents pour le comportement d'achat a. Assurez coopératives avec environ un tiers de la production de vin d'une partie importante du marché. En raison de la structure d'une coopérative, mais il ya des obstacles de l'image de marque, qui ne permet pas les investissements stratégiques à long terme dans la construction d'une marque. Nouvelle économie institutionnelle est souvent utilisé dans la recherche agronomique explication pour les agriculteurs dans les coopératives sont axés sur la maximisation du prix de rachat et donc rejettent investissements stratégiques. Depuis les vignerons sont tributaires de la coopération avec sa base de membres en raison du principe de la démocratie, par conséquent, peut être omis investissements stratégiques nécessaires. Ce document fournit des explications pour les déficits dans les entreprises stratégiques coopératives de transformation dans l'industrie du vin et cherchent à trouver des solutions.

\section{Einleitung}

Genossenschaften sind eine wichtige Rechtsform in der Weinwirtschaft. Circa 32.000 ha Weinberge werden in allen 13 deutschen Anbaugebieten genossenschaftlich bewirtschaftet - mit dieser Fläche werden durchschnittlich etwa 3 Mio. hl Wein erzeugt (DRV, 2011). Rund ein Drittel der deutschen Weinerzeugung wird in Deutschland von genossenschaftlich organisierten Betrieben verantwortet. In den einzelnen Anbaugebieten haben Winzergenossenschaften eine noch größere Bedeutung. In Baden und Württemberg dominieren die Winzergenossenschaften mit fast 70 Prozent den Anbau (Weinwirtschaft, 2013a). Im Anbaugebiet Saale-Unstrut nimmt die Winzervereinigung Freyburg-Unstrut sogar die Hälfte der Rebfläche des Anbaugebiets ein und trägt damit als Einzelbetrieb eine herausragende Rolle. 179 Winzer- und Weingärtnergenossenschaften existieren zurzeit in Deutschland (DRV, 2011). Diese - allerdings rückläufige - Anzahl der Genossenschaften verdeutlicht, dass durch Fusionen und Kooperationen die Wirtschaftlichkeit und damit die Wettbewerbsfähigkeit kontinuierlich verbessert werden, um die Marktstellung der deutschen Winzergenossenschaften zu erhalten.

This is an Open Access article distributed under the terms of the Creative Commons Attribution License 4.0, which permits unrestricted use, distribution, and reproduction in any medium, provided the original work is properly cited. 
Erfolge bei nationalen und internationalen Prämierungen sind der Beweis für das hohe Qualitätsstreben der genossenschaftlichen Weinbaubetriebe. So wurden beispielsweise 98,5\% der getesteten deutschen Genossenschaftsweine von der Weinwirtschaft im Jahr 2013 mit "sehr gut" oder "gut" bewertet (Weinwirtschaft, 2013b). Qualität ist auf dem wettbewerbsintensiven deutschen Weinmarkt allerdings kein Garant mehr für konstante oder steigende Marktanteile. Gerade im LEH bietet sich eine Produktdifferenzierung über den Aufbau einer Marke an, da hier - anders als im Fachhandel oder "ab-Hof" keine beratende und einflussnehmende Kraft auftritt und damit der Kunde orientierungslos am Weinregal zurückgelassen wird. Winzergenossenschaften haben gute Voraussetzungen für den Verkauf ihrer Weine über den Lebensmitteleinzelhandel. Im Vergleich zu selbstvermarktenden Weingütern, haben Genossenschaften meist die Quantitäten um den überregionalen LEH zu beliefern, und Weine deutscher Kellereien werden meist weniger prominent im Regal platziert. Für die Abnehmer sind die Winzergenossenschaften des Weiteren vertrauenswürdige Lieferanten, die sich der Qualität verpflichten.

Die genannte Strategie der Markenführung verlangt nach langfristig angelegten strategischen Investitionen, die in einer Genossenschaft durch die Mitglieder getragen werden müssen. Allerdings scheuen viele Winzergenossenschaften Investitionen in langfristige Strategien, die die Wertschöpfung steigern könnten, wie den eben genannten Markenaufbau oder auch die Internationalisierung oder Marktsegmentierung (Schramm et al., 2004). Oft scheitern entsprechende Versuche in den genossenschaftlichen Gremien am Widerstand der Landwirte (Wocken et al., 2008). Die Strukturen der Rechtform Genossenschaft, stehen dabei oft den Investitionen in langfristigen Strategien im Weg. Hieraus resultieren Probleme zahlreicher Genossenschaften, langfristige Investitionen in eine immaterielle Marke zu tätigen und spezifisches Vermögen aufzubauen. Somit ist das Ziel dieses Papers, Erklärungsansätze für strategische Defizite in genossenschaftlichen Verarbeitungsunternehmen der Weinindustrie zu liefern und einen Lösungsansatz anzusprechen.

\section{Markenverständnis}

Das Markenverständnis wie wir es heute kennen, als idealerweise identitätsorientiertes Markenverständnis, hat sich erst seit den 90er Jahren herausgebildet. Seit der Entstehung des klassischen Markenartikelkonzeptes haben Änderungen in den Markt- und Umweltbedingungen das Verständnis vom Wesen einer Marke tiefgreifend gewandelt. Zunächst war, Mitte des 19. Jahrhunderts mit Einsetzen der Industrialisierung das Markenverständnis durch den bloßen Vorgang der Kennzeichnung geprägt. Markenführung als betriebswirtschaftliches Managementkonzept existierte nicht (Meffert und Burmann, 2005). In der zweiten Entwicklungsphase war das Verständnis vom Wesen der Marke durch einen konsumgüterorientierten Warenfokus und der damit verbundenen Suche nach konstitutiven Eigenschaften verbunden. Der Markenbegriff wurde durch einen Merkmalskatalog gekennzeichnet
(Tomczak et al., 2005). Das angebotsorientierte Markenverständnis der dritten Entwicklungsphase ab Mitte der 60er Jahre war stark an Produktion- und Vertriebsmethoden orientiert (Dichtl 1978). In der vierten Entwicklungsphase, etwa ab Mitte der 70er Jahre bis Ende der 80er, waren die Rahmenbedingungen geprägt durch zunehmende Sättigungstendenzen auf vielen Märkten (Meffert und Burmann, 2005). Das Markenverständnis war von einer nachfragebezogenen, subjektiven Begriffsauffassung geprägt. Nur die Produkte, waren als Markenartikel zu bezeichnen, welche auch von den Konsumenten als solche wahrgenommen wurden (Berekoven 1987; Meffert 1979). Die letzte Entwicklungsphase ab Anfang der 90er Jahre ist von einer Angleichung der Produktqualitäten gekennzeichnet. Auf Basis der vorhergehenden Ansätze hat sich das identitätsorientierte Markenverständnis herausgebildet. Im Rahmen dieses Verständnisses wird die Relevanz von Marken für das Kaufverhalten hauptsächlich auf deren Identität zurückgeführt. Eine konsistente Markenidentität bildet dabei die Voraussetzung für das Vertrauen der Nachfrager in die Marke (Meffert und Burmann, 2005).

Im identitätsorientiertem Markenmanagement konstituiert sich das Nutzenbündel Marke aus materiellen und immateriellen Komponenten in zweifacher Weise. Zum einen aus physisch-funktionalen Nutzenkomponenten und zum anderen aus symbolischen Nutzenkomponenten. Diese können in unterschiedlicher Weise zur nachhaltigen Differenzierung und damit zur Entstehung und Stärke einer Marke beitragen. Somit wird häufig auf die die nutzenorientierte Wirkungsweise von ESCH verwiesen. Nach ESCH (2000, S.11) liefert eine Marke:

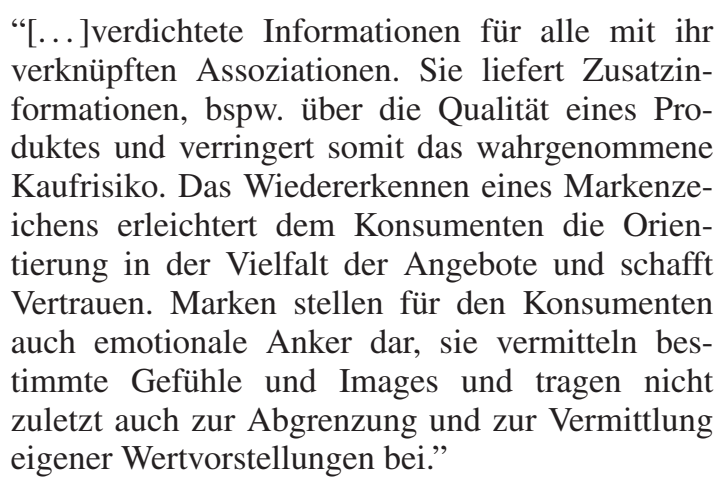

Eine Marke senkt also das Kaufrisiko in dem Vertrauen zum Konsumenten aufgebaut wird. Außerdem dient eine Marke im Regal zur schnellen Wiedererkennung und generiert ein hohes Nutzenversprechen in Form von spezifischer Qualität und spezifischen Wertevorstellungen (Orth und Malkewitz, 2008). Als firmenspezifisches Element der Abgrenzung ermöglichen Marken die Unterscheidung von vergleichbaren Produkten in einer ähnlichen Kategorie. Marken sind folglich Träger von Bedeutungen und werden auf komplexe Weise und auf verschiedenen Ebenen vom Konsumenten wahrgenommen. Entscheidend aber ist, dass die Markenmacher und die Konsumenten, der Marke dieselbe Bedeutung zuschreiben. 


\section{Die Bedeutung von Markenweinen in der deutschen Weinwirtschaft}

Um sich im Weinmarkt, einem Markt mit hoher Wettbewerbsintensität durchzusetzen, bedarf es der Produktdifferenzierung. Somit gewinnt Markenbildung in der Weinwirtschaft immer mehr an Bedeutung (Hanf und Schweickert 2014; Weinwirtschaft 2012). Betrachtet man die Kategorie Leistungspolitik von weinproduzierenden Unternehmen, so haben diese, innerhalb dieser mit dem Markenaufbau ein Mittel, um sich mit ihren Produkten von den Konkurrenten zu differenzieren (Orth und Malkewitz, 2008). Welche Alternativen hätte ein weinproduzierendes Unternehmen? Weine sind in der Regel regionale Produkte, deren Qualität nach dem Verständnis der meisten Konsumenten ausschließlich durch Rebsorte, Herkunft und Jahrgang geprägt werden (Hoffmann und Stumm, 2007). Sich sensorisch oder auch Qualitativ von seinem Konkurrenten abzusetzen, gestaltet sich in der heutigen Zeit schwierig, da sich in den letzten Jahrzehnten ein konstantes und verlässliches Niveau etabliert hat. Grob fehlerhafte Weine findet man so gut wie nicht mehr und auch geschmacklich haben sich verschiedene (meist einfach gehaltene) Profile durchgesetzt in denen jeder Käufer sich seiner Präferenz nach wiederfindet. Besonders im Hinblick auf die, zum größten Teil sensorische Laienhaftigkeit der Konsumenten, ist von einer Differenzierung über ein "besonderes" Geschmacksprofil abzuraten.

Unter Winzern oft verbreitet ist die Annahme, spezielle Lagen bieten eine Abgrenzungsmöglichkeit. Auch dieses Argument lässt sich entkräften, über zum einen der Tatsache, dass viele Lagen nicht in Alleinbesitz sind und dem wichtigeren Fakt, dass der gemeine Konsument auch dazu wieder nicht über die nötige Fachkompetenz verfügt. Nicht zuletzt wurden viele Lagen erst bekannt durch den Namen und die Produkte eines Weingutes und nicht andersherum. Auch die Literatur verweist darauf, dass sich Lagen nicht zur Differenzierung und Profilierung eignen. Auch neue innovative Produkte sind in der Weinwirtschaft nicht mehr einfach etablierbar, da sich bereits viele Produzenten mit den unterschiedlichsten Produkten auf dem Markt befinden und das Produkt Wein nur ein begrenztes Innovationpotenzial bietet, welches bereits nahezu ausgeschöpft ist. (Göbel, 2010)

Eine Alternative, zur Markenbildung im Produktbereich bietet die Unternehmerpersönlichkeit. Eine Abgrenzung über die Persönlichkeit des Winzers ist nicht immitierbar, i.d.R. authentisch und wird von zahlreichen Weingütern praktiziert, da diese Form sehr guten Anklang bei den Kunden findet (Göbel, 2010). Allerdings eignet sich dazu eine Winzergenossenschaft strukturell bedingt nur sehr begrenzt. Der Kopf einer Genossenschaft ist ein angestellter Geschäftsführer. In einem klassisch familiengeführten Weingut ist die Unternehmerpersönlichkeit der Winzer oder Kellermeister - ein Familienmitglied und als solches im Betrieb verwachsen. Dieser Umstand bewirkt eine wesentlich authentischere Ausstrahlungskraft als identitätswirkende Persönlichkeit.

Es wird also deutlich, dass nur der Aufbau einer Marke die Chance bietet, sich als Genossenschaft eineindeutig zu profilieren und letztlich erfolgreich zu differenzieren. Aus Sicht der Genossenschaft birgt die Marke noch weitere Folgenutzen, wie eine Wertsteigerung des Unternehmens, Kundenbindung, eine segmentspezifische differenzierte Marktbearbeitung und ein erweiterter preispolitischer Spielraum. Zudem erhöht es den Wiedererkennungswert des Produktes enorm. Auch für den Verbraucher der Orientierung, Sicherheit und Komfort beim Weineinkauf sucht, bieten sich die Markenweine an. Denn, eine Marke senkt das Kaufrisiko und bietet dem Verbraucher zugleich bestimmte Nutzen wie Vertrauensbildung, spezifische Qualität und spezifische Wertevorstellungen die mit der Marke verbunden sind.

\section{Erklärungsansätze für strategische Probleme der Markenführung}

Die Marke ist das Ergebnis einer Vielzahl über einen längeren Zeitraum durchgeführter Maßnahmen und der hierauf basierenden Erfahrungen der Nachfrager. Der Managementprozess dieser Maßnahmen umfasst dabei Planung, Koordination und Kontrolle (Tomczak et al., 2005). Um die Markenbildung erfolgreich umzusetzen, bedarf es umfangreicher Lernvorgänge. Damit ist das systematische Ändern des Verhaltens aufgrund von Erfahrungen gemeint (Burmann et al., 2005). Die Nachfrager müssen das in der Positionierung zum Ausdruck kommende Nutzenversprechen einer Marke lernen. Es muss verinnerlicht werden, worin im Vergleich zu konkurrierenden Produkten der Mehrwert der Marke liegt, und schließlich wo die Marke zu erwerben ist. Bei der Markenentwicklung ist darauf zu achten, dass Lernen mit hohem Zeitbedarf einhergeht. Um eine erfolgreiche Markenführung umzusetzen, bedarf es folglich eines hohen Maßes an Geduld beziehungsweise eines langen Planungshorizontes (Weinberg, 2003).

Somit ergeben sich bei dieser Art der Wertschöpfungsstrategien bei Genossenschaften Probleme bezüglich der Verteilung der Verfügungsrechte, da zunächst über mehrere Jahre umfangreiche Investitionen erfolgen müssen (Fulton, 1995). Die Mitglieder einer Winzergenossenschaften haben in der Regel aus ökonomisch nachvollziehbaren Gründen wenig Interesse an einer Steigerung des Unternehmenswertes durch strategische Investitionen, da sie nach dem Genossenschaftsgesetz kein Recht auf Erträge, welche aus einer Steigerung des Unternehmenswertes resultieren (Frick, 2004). Ursachen sind die fehlende Verzinsung der Genossenschaftsanteile und die Abwesenheit eines Marktes für diese, da Genossenschaftsmitglieder beim Ausstieg aus der Genossenschaft ihre Anteile nicht verkaufen, sondern nur zum Nennwert zurückgeben können. Eine unzureichende Spezifizierung der Verfügungsrechte führt folglich zu einer Fokussierung der Winzer auf einen kurzfristigen Auszahlungspreis, sowie $\mathrm{zu}$ einer ungenügenden Anreizgestaltung für strategische Investitionen, so dass diese nicht von den genossenschaftlichen Anteilseignern mitgetragen werden (Dilger, 2005). Nun ist aber nicht nur von Bedeutung wie vollständig die Verfügungsrechte zugeordnet sind, sondern auch wie viele Träger dieses Recht hat. Umso höher die Anzahl der Verfügungsrechtsträger, umso verdünnter 
sind die Verfügungsrechte (Frick, 2004). In einem inhabergeführten Weingut gibt es nur einen oder wenige von denen die Verantwortung getragen wird und welche von einem unterstellten Unternehmenswertzuwachs profitieren würden. Eine Genossenschaft allerdings hat viele stimmberechtigte Mitglieder, welche gleichzeitig Anteilseigner sind. Die Verfügungsrechtestruktur der Winzergenossenschaftsmitglieder kann daher als sehr verdünnt bezeichnet werden (Fulton, 1995).

Ziel der Markenentwicklung ist es eine Steigerung des ökonomischen Markenwertes herbeizuführen. Der ökonomische Mehrwert kann dabei auf zwei unterschiedlichen Faktoren beruhen. Zum einen wird der Mehrwert häufig operationalisiert über die Preisprämie, die ein Nachfrager für ein Markenprodukt im Vergleich $\mathrm{zu}$ einem markenlosen Produkt $\mathrm{zu}$ zahlen bereit ist (Sander 1994, Schweiger 1998). Zum anderen kann der Markenwert sich auch in der Absatzmenge niederschlagen, wenn der wahrgenommene Nutzenvorteil einer Marke in Abwägung gegenüber preisgleichen markenlosen Produkten überwiegt. Beides tritt in der Praxis häufig als Kombination auf.

In diesem Kontext kann ein weiteres Problem, das Trittbrettfahrer-Problem, bei Genossenschaften auftreten (Cook, 1995). Das Genossenschaftsgesetz verbietet eine zeitliche Diskriminierung der Mitglieder, so dass neu hinzukommende Mitglieder den gleichen Auszahlungspreis für ihre Trauben erhalten wie langjährige Mitglieder. Wenn Letztgenannte aber im Zuge einer Markeninvestition auf Rendite verzichtet haben, sollte ihnen ein höherer Auszahlungspreis gewährt werden können (Frick, 2004). Dies führt erneut zu einer Orientierung an der kurzfristigen Maximierung der Auszahlungspreise, da sie die einzige Möglichkeit zur Amortisierung der getätigten Investitionen darstellt. Die grundsätzlich im Genossenschaftsgesetz vorgesehene anteilige Auszahlung eventuell erwirtschafteter Unternehmensgewinne an die Genossen, wird zum Ende eines jeden Geschäftsjahres vorgenommen (Pennerstorfer und Weiss, 2013). Somit ist diese erneut am kurzfristigen Unternehmenserfolg orientiert und bietet wieder keinen Anreiz langfristigen Investitionsstrategien zum Markenaufbau zu verfolgen. Letztendlich muss festgehalten werden, dass wenn kurzfristig zu realisierende Erfolge im Mittelpunkt des Management- oder Mitgliederinteresses stehen, verkommt Markenführung schnell zur "Markierung" und die Wirkung der Marke verpufft (Burmann et al., 2005).

Zum anderen ist auch der Planungshorizont (HorizontProblem) der Mitglieder der Winzergenossenschaft heterogen (Cook, 1995). Speziell die älteren Genossen und die deren Nachfolge nicht klar geregelt ist unterliegen diesem Problem, welches dazu führt langfristig angelegte Investitionen nicht zu unterstützen. Die Wahrscheinlichkeit, dass die Genossen im Rahmen ihrer verbleibenden Mitgliedszeit nicht mehr an den Erfolgen der Investitionsentscheidungen partizipieren können, ist groß (Dilger, 2005). Vielmehr werden sie eine Maximierung der Auszahlungspreise fordern. Gerade von Winzergenossenschaften mit einem hohen Anteil an Genossen in der Endphase der Mitgliedschaft sind daher kaum Impulse für strategische Investitionen zu erwarten (Schramm et al.,
2004). Hinzukommt, dass wenn man die Struktur vieler Winzergenossenschaften bezüglich Haupt- und Nebenerwerbsmitgliedern betrachtet, einem auffällt, dass es in zahlreichen Winzergenossenschaften viele Nebenerwerbswinzer gibt. Im Gegensatz zu der Minorität der vollerwerblichen Traubenproduzenten betreibt die mehr oder weniger überwiegende Zahl an Nebenerwerbswinzern den Weinbau mehr als Hobby und Liebhaberei, als zum Lebensunterhalt. Aufgrund dieser Heterogenität der Mitglieder kann die diskutierte Investitionsproblematik noch verschärft werden (Hanf und Schweickert, 2007).

Die Zielkonflikte der langfristigen Strategieausrichtung der Geschäftsführung zum einen und der kurzfristigen Nutzenmaximierung der Genossenschaftsmitgliedern zum anderen lässt sich auch auf das aus der Literatur bekannte Prinzipal-Agenten-Dilemma zurückführen. Prinzipal und Agent verfügen hierbei über unterschiedliche Nutzenvorstellungen (Dilger, 2005). Der Prinzipal verhält sich als rationaler Nutzenmaximierer und handelt damit nicht mehr zugleich im besten Interesse des Prinzipals. Auf der anderen Seite können die Mitglieder nicht immer beurteilen, ob die Manager die beste Investitionsalternative treffen, oder ob eigennützige Aspekte wie Machtzuwachs oder Statusgewinn ausschlaggebend sind (Sykuta und Cook, 2001). Festzuhalten bleibt, dass sich die Eigentümer der Winzergenossenschaft nicht sicher sein können, dass das Management hinreichend qualifiziert (Qualitätsrisiko) und gewillt ist (Moral-Hazard-Risiko), die Geschäfte der Genossenschaft ausschließlich im Interesse der Eigentümer zu führen, (Chaddad und Cook, 2004).

\section{Diskussion und ausblick}

Einen Lösungsansatz der angesprochenen Probleme liefern die aus den USA bekannten New Generation Cooperatives (NGC's) (Cook und Chaddad 2004). Eine NGC ist eine Art Genossenschaft, die ein System der Lieferrechte und -pflichten verwendet, um Geschäftsbindung $\mathrm{zu}$ fördern und eine Form der vertikalen Integration anbietet (Harris et al., 1996). NGC's sind besonders geeignet für landwirtschaftliche Unternehmen auf der Wertschöpfungsebene der Verarbeitung und Vermarktung. Manchmal werden sie auch als Hybriden zwischen traditionellen Genossenschaften und Aktiengesellschaften beschrieben. NGC's könnten ein Weg sein, die Lücke zwischen rohstofforientierten Primärproduzenten und den verbraucherorientierten Verarbeitungsunternehmen zu schließen (Alberta, 2014; Van Dyjk 1997).

NGC's können an die Ausgabe von Genossenschaftsanteilen an Lieferrechte und -pflichten binden. Mitglieder haben die Möglichkeit ihre Genossenschaftsanteile durch Zukauf dieser zu erhöhen, wodurch sich für die Genossen wiederrum der Anreiz für langfristig angelegte Investitionen erhöht. Ferner kann die Mitgliedschaft begrenzt werden auf festgelegte Anteilseigner (Cook 1997). Durch diese spezifischere Ausgestaltungen der Verfügungsrechte und den incentive-basierten Ansätzen der Auszahlungsmodalitäten, lassen sich gegebenenfalls die Probleme der langfristigen strategischen Investitionen der klassischen Genossenschaften lösen oder zumindest vermindern. Zeitverzögerte Auszahlungsmöglichkeiten, 
umgelegte Einzahlungsbeiträge welche im Sinne eines Generationenvertrages ausgezahlt werden, oder auch erfolgsabhängige, an den Markenwert gekoppelte Auszahlungssysteme, würden Schranken klassischer Genossenschaften überwinden (Harris et al., 1996). Drescher und Ratjen (1999) haben gezeigt, dass in Deutschland grundsätzlich der Ansatz der NGC's umsetzbar wäre.

Die Entscheidungen der Winzer folgen jedoch möglicherweise nicht immer dem im Property-Rights-Ansatz unterstellten streng rationalen Ziel der Gewinnmaximierung, sondern hängen von weiteren psychologischen Faktoren wie z. B. Zufriedenheit oder Risikobereitschaft ab. Das tatsächliche Verhalten weicht umso stärker vom erwarteten Verhalten ab, je komplexer die Entscheidungssituation ist (Simon, 1959; Pfohl und Braun, 1981). Die Bewertung einer strategischen Investition in den Aufbau einer Marke stellt so eine mit hohen Risiken behaftete und schwierig kalkulierbare Entscheidungssituation dar. Die Investitionsbereitschaft in eine Wertschöpfungsstrategie lässt sich daher vermutlich nicht allein mit der Ausgestaltung der Property-Rights erklären, sondern wird von weiteren Faktoren beeinflusst. Die verhaltenswissenschaftlichen Ansätze würden hier weiteren Raum zur Betrachtung und Diskussion des Themas liefern.

\section{Literatur}

Alberta (2014): http://www1.agric.gov.ab.ca/ \$department/deptdocs.nsf/all/bmi6646.

Abgerufen am: 14.02.2014

Burmann, C., Meffert, H., Koers, M. (2005): Stellenwert und Gegenstand des Markenmanagements, in: Meffert, H., Burmann, C., Koers, M. (Hrsg.): Markenmanagement, Identitätsorientierte Markenführung und praktische Umsetzung, 2. Auflage, Gabler Verlag

Chaddad, F.R. und Cook, M.L. (2004): "Understanding New Cooperative Models: An Ownership-Control Rights Typology", Review of Agricultural Economics, 26: $348-360$

Cook, M.L und Chaddad, F.R. (2004): Redesigning Cooperative Boundaries: The Emergence of New Models. American Journal of Agricultural Economics, 86 (5): 1249-1253

Cook, M.L. (1997): “Organizational Structure and Globalization: The Case of User Oriented Firms" in Nilson, J. and van Dijk, G. (eds.) "Strategies and Structures in the Agro-food Industries", van Gorcum: 77-93

Cook, M.L. (1995): The Future of U.S. Agricultural Cooperatives: A Neo-Institutional Approach. In: American Journal of Agricultural Economics 77 (5): 1153-1159

Dichtl, E. (1978): Grundidee, Entwicklungsepochen und heutige wirtschaftliche Bedeutung des Markenartikels, in: Gabler Verlag (Hrsg.), Markenartikel heute. Marke, Markt und Marketing, Wiesbaden, S. 17 - 34

Dilger, A. (2005): "Prinzipal-Agenten-Probleme im deutschen Weinbau". In: Zeitschrift für das gesamte Genossenschaftswesen 55 (3): 179-189
DRV (Deutscher Raiffeisenverband) (2011): http:// www.raiffeisen.de/uebersicht-der-genossen schaftssparten/wein/. Abgerufen am: 10.01.2014

Esch, R.R. (2000): Herausforderungen und Aufgaben des Markenmanagements. Moderne Markenführung, Gabler Verlag, Wiesbaden

Fulton, M. (1995): "The Future of Canadian Agricultural Cooperatives: A Property Rights Approach". American Journal of Agricultural Economics, 77: 1144-1152

Frick, B. (2004): Does Ownership Matter? Empirical Evidence from the German Wine Industry. In: KYKLOS, 57, 3, 357-386

Göbel, R. (2010): Das Weinsortiment mit eigenem Profil zum Verkaufserfolg, DLG-Verlag, Frankfurt am Main

Hanf, J.H. und Schweickert, E. (2014): Cooperatives in the balance between retail and member interests: The challenges of the German cooperative sector, Journal of Wine Research. 25, Nr. 1; S. 1-13

Hanf, J.H. und Schweickert, E. (2007): "How to Deal with Member Heterogeneity - management implications". In: International Journal of Co-operative Management 3 (2): 40-48

Harris, A., Stefanson, B. und Fulton, M. (1996): New generation cooperatives and cooperative theory. Journal of Cooperatives, 11: 15-22

Hoffmann, D. und Stumm (2007): Marken und geographische Angaben bei Qualitätsweinen aus Rheinland-Pfalz, Forschungsanstalt Geisenheim

Meffert, H. und Burmann, C. (2005): Wandel in der Markenführung - vom instrumentellen zum identitätsorientierten Markenverständnis, in: Meffert, H., Burmann, C., Koers, M. (Hrsg.): Markenmanagement, Identitätsorientierte Markenführung und praktische Umsetzung, 2. Auflage, Gabler Verlag

Orth, U.R. und Malkewitz, M. (2008): Holistic Package Design and Consumer Brand Impressions. Journal of Marketing, 72 (3): 64-81

Pennerstorfer, D. und Weiss, C.R. (2013): Product quality in the agri-food chain. Do cooperatives offer highquality wine? In: European Review of Agricultural Economics, 40 (1): 143-162

Pfohl, H.-C. und G. Braun (1981): Entscheidungstheorie, Normative und deskriptive Grundlagen des Entscheidens. Moderne Industrie, Landsberg am Lech

Sander, M. (1994): Die Bestimmung und Steuerung des Wertes von Marken. Eine Analyse aus Sicht des Markeninhabers, Heidelberg

Schramm, M., Taube, M. (2004): Privat Ordering of Corrupt Transactions: The Case of Chinese GuanxiNetworks and Their Challenge by a Formal Legal System, in: Lambsdorff, J., Schramm, M., Taube, M. (Hrsg.): Corruption and the New Institutional Economics, London, New York

Schweiger, G. (1998): Markenwertforschung: Die Konsumenten bestimmen den Wert einer Marke, in: Werbewissenschaft und Marktforschung, Leistungsbericht, Wien, S. 20-23 
Simon, H. (1959): Theories of decision-making in economics and behavioral science. In: The American Economic Review 49 (3): 253-283

Sykuta, M.E. und Cook, M.L. (2001): “A New Institutional Approach to Contracts and Cooperatives". American Journal of Agricultural Economics, 83: 12731279

Tomczak, T., Schögel, M. und Feige, S. (2005): "Erfolgreiche Markenführung gegenüber dem Handel", 4. Edition, Gabler Verlag, Wiesbaden, Germany

Van Dijk, G. (1997): "Implementing the Sixth Reason for Co-operation: New Generation Co-operatives in Agribusiness" in Nilson, J. and/van Dijk, G. (eds.) "Strategies and Structures in the Agro-food Industries", van Gorcum: 94-110

Weinberg, P. (1993): Markenartikel und Markenpolitik, in: Wittmann, W.; Kern, W.; Köhler, R.; Küpper,
H.-U.; v. Wysocki, K. (Hrsg.), Handwörterbuch der Betriebswirtschaft, Teilband 2 (I-Q), 5., völlig neu gestaltete Aufl., Stuttgart, S. 2679-2690

Weinwirtschaft (2013a): Deutsche Winzergenossenschaften, Leuchttürme der Region in: Weinwirtschaft Ausgabe 14l13 vom 5. Juli 2013, S. 28

Weinwirtschaft (2013b): Leistungstest deutsche Genossenschaften in: Weinwirtschaft Ausgabe 14l13 vom 5. Juli 2013, S. 48

Weinwirtschaft (2012): Markenweine - Leuchttürme im Regal. in: Weinwirtschaft Ausgabe 21112 vom 12. Oktober 2012, S. 19

Wocken, C., Hemme, T., Ramanovich, M., Fahlbusch, M. und A. Spiller (2008): Der Markt für Milch und Milcherzeugnisse. In: Agrarwirtschaft 57 (1), Supplement: Die landwirtschaftlichen Märkte an der Jahreswende 2007/2008: S. 36-58 